\title{
MULTIDISCIPLINARY ISSUES IN MARINE SCIENCE
}

\author{
By Donald B. Olson
}

\section{The picture}

of ocean science as

the home of diversely

trained scientists

working in unison on

multidisciplinary issues

is not completely

accurate.
B

EFORE considering the actual state of multidisciplinary efforts in the field of marine science today, it is worth contemplating the reasons why multidisciplinary interaction is so important to oceanography and related fields of marine research. It is customary to offer multidisciplinary cross-fertilization and cooperation as a panacea for stagnated productivity and performance in U.S. research and development efforts. However, the rationale for such interaction should be more extensive in marine science; in many ways is part of the history of the field.

The reasons used to justify support of marine science in relation to priorities in other fields can be consolidated in three areas:

- The role the ocean plays in resources and their depletion (i.e., oil production, pollution, fisheries. medical research, and recreational use),

- Aspects involving use of the ocean for commerce and military transport (i.e.. ship routing and safety. environmental aspects of maintaining a Navy at sea. and means for using it effectively in wartime), and

- The oceans" role in the Earth's climate system (i.e.. weather prediction, long term climate prediction, and planning for the impact of climate change).

Each of these is tied to multidisciplinary concerns, even if. in some cases. they are the province of single groups of experts.

Overall there is a healthy interaction between biologists, chemists, physicists and geologists involved in oceanography. These interactions are fostered by the nature of the organization of the institutions where ocean research is carried out, in the graduate training many scientists receive, and in the realities of carrying out research at sea. Most ocean science today takes place in oceanographic institutions, gov'ernment laboratories, or in oceanography departments at various universities. In the largest of these. there are often internal division that encompass biology, chemistry, physics and geology. The academic heritage of many marine scientists is tilted at least in numbers to the large, reasonably complete programs. At sea, even in cases where cruises are primarily planned for activities in a single discipline, it is

Donald B. Olson. Rosenstiel Scherd of Marine and Amespherte Silences. University of Miami. FL 33149. common to have one or two others on board to complete sampling in other disciplines. There have been a number of genuinely multidisciplinary experiments, such as the Coastal Upwelling Experiment, or the Warm-Core Rings program, in which science efforts were planned, carried through, and in a few cases, published with true cooperation between disciplines.

The picture of ocean science as the home of diversely trained scientists working in unison on multidisciplinary issues is not completely accurate. Perhaps the least cross-field interaction occurs in marine medical studies, basic fluid dynamics, and fisheries research. In each of these areas, practitioners must spend considerable time keeping abreast of developments outside oceanography. In the case of medical research, this is perhaps understandable when the principal activity is identification and collection of organisms for laboratory purposes that may have little relation to the ocean. In fluid mechanics, many theoreticians interact regularly with their field-and laboratory-oriented colleagues, but overall the realm of theoretical fluid dynamics is more closely tied to the fields of pure physics, applied math, or allied disciplines such as meteorology.

The small amount of interaction in the fisheries field is more difficult to understand. Fisheries research is by its very nature multidisciplinary, because it combines management goals with fish biology. While this has led to a fairly broad interaction in statistics, economics, and the population dynamics of fisheries biology, fisheries science has in many respects not benefitted fully from recent advances in biological and physical oceanography. A portion of this estrangement may be a result of the organization of some fisheries institutions, which sometimes are separate entities within a university or a completely separate government laboratory. In such cases, crossdisciplinary lines of communications in fisheries tend to be more with colleagues in statistics or management than with oceanographers.

Upon close inspection, some areas of active multidisciplinary research in ocean science reveal little cooperative interaction. Climate related issues, for example, are of interest to perhaps the largest spectrum of ocean scientists. Here the trend is toward 
groups in each subdiscipline that work in their own areas. Most of the interaction between, say, the geochemistry and the geophysical dynamics community is through the literature, rather than by means of a concentrated attempt to establishment multidisciplinary efforts. Despite these shortcomings. the oceanographic community has maintained much interdisciplinary communication, and in many cases. true multidisciplinary work. The field of ocean science has in many ways pioneered large multidisciplinary science, while on the other hand it has exemplified the major impediments to and needs for such efforts.

One aspect of the problem is the source of funding for multidisciplinary efforts. Support of multidisciplinary work presents several difficult issues to which there are probably no concrete solutions. Within some portions of the community, the primary role of the laboratories involved may provide institutional barriers. For example, a laboratory charged with regional fisheries management may require information on biomedical problems or ocean circulation questions, but this information may lie outside the range of expertise established by the charter of the laboratory. The involvement of such groups in multidisciplinary approach clearly demands the identification of the source for the additional expertise to make up a research team. Managing disparate groups set in different laboratory environments produces further difficulties. The worst situation occurs when an outside group is tapped to start an effort but in the end most of the resources are spent locally, with little use of the outside resource at the crucial stage of doing the science.

The situation in university settings can be almost as daunting. Here the problem is not the identification of participants, since there are few boundaries to preclude interaction between academic scientists across the country. Rather the difficulties occur at the point of obtaining the necessary funding. Unless the purpose is simply the addition of a single investigator from an outside field for a particular service to the program, a multidisciplinary effort will be scrutinized by more than one discipline as part of the review process. Therefore a joint physical-biological program will be reviewed by both physical and biological oceanographers for its content in these areas, as well as for its multidisciplinary aspects. In general, this is appropriate since it makes little sense to put together a program in which one of the components is not at least competent in its own field. When it comes to actual review. most of the federal agencies have developed means for carrying out this process in a fair way. The nagging question to be faced is what to do when the science is competently proposed. but one of its disciplinary areas lies outside the interests of one of the fields? In current budgetary climates how does one view the expenditure of resources from one sub-field for programs where the expected advance in knowledge will be in another field? This single question is at the heart of the multidisciplinary nature of ocean sciences.

Within the academic community, a related issue is required course work outside distinct specialties. Even a cursory examination of marine science programs suggests that there are differing ideas about how much multidisciplinary education is needed to produce a marine scientist. At the graduate level, one philosophy is to begin the graduate career with a set of overview courses taught to either the entire incoming class or specifically to each non-major group; in such an arrangement biologists, for example, get a survey course in physical oceanography or chemistry but take a more concentrated introduction in biological oceanography. In many programs the whole issue is ignored. and students in certain fields (e.g., physical oceanography at the University of Miami) students may never receive a formal introduction to the breadth of ocean science.

There are some instances where the ignorance of the other fields in oceanography is held up at least unofficially as a virtue. This attitude disregards the basic nature of ocean studies as a multidisciplinary activity, and it is unhealthy for ocean science as an organized field. The academic pressures for mastery of the specialty versus the broad introduction to the field, which are the source of these feelings, are not easily dismissed. In some cases, educational programs that attempt to produce the best possible physicist, biologist or chemist are at odds with the "core course" concept, because the three or four required core classes compete with the time required to instruct a student in specialty subjects. However, when the specialist mentality prevails, sometimes an overly narrow perspective results; for example, a physical oceanographer who is at a loss about plankton, or a marine biologist with no concept of mixing processes and water masses.

In conclusion, ocean sciences as a whole can be proud of the degree to which multidisciplinary themes stand at the heart of our profession. In many ways oceanography is an example of how multidisciplinary research can be accomplished. However, there remain difficult questions about how to strengthen the interactions between the various components of marine science, now and in the future.

The several multudsciplinary oceanographic research programs mentioned above studied interactions among physical, bio logical. chemical and geological processes in a variety of marme environments. Interdisciplinary mathematical models represent synthesis tools that blend theory and observations to aid in understanding and analyzing the diverse data sets that result from these oceanographic studies. Interdisciplmary modelıng is expected to play an important role in designing sampling strategies for and interpreting data collected during the large multidisciplinary research programs planned for the coming decade. Consequently, interdisciplinary modeling is an exciting and developing research area and an especially fertile area for new students entering the oceanography profession. Hopefully the number of students trained in modeling techniques will increase in the coming years. However, this will require that the traditional curriculum be moditied to give students the flexibility to obtain training in areas outside of a particular oceanographic discipline as well as training in applied mathematics.

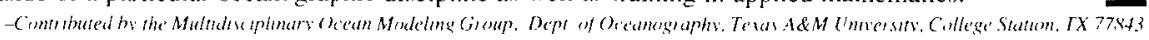

\section{One aspect}

of the problem is the

source of funding for

multidisciplinary efforts. 